\title{
A Multi Layer Evidence Network Model for the Design Process of Space Systems under Epistemic Uncertainty
}

\author{
Gianluca Filippi * \\ Early Stage Researcher, Aerospace Centre of Excellence, Mechanical and Aerospace Engineering \\ University of Strathclyde, James Weir Building, 75 Montrose Street, Glasgow, UK \\ Email: g.filippi@strath.ac.uk \\ Massimiliano Vasile \\ Professor, Aerospace Centre of Excellence, Mechanical and Aerospace Engineering \\ University of Strathclyde, James Weir Building, 75 Montrose Street, Glasgow, UK \\ Email: massimiliano.vasile@strath.ac.uk
}

\begin{abstract}
Summary
The purpose of this paper is to introduce a new method for the design process of complex systems affected by epistemic uncertainty. In particular, a multi-layer network is proposed to model the whole design process and describe the transition between adjacent phases. Each layer represents a design phase where each node is a subsystem and each link a sharing of information. The network is used to quantify and propagate uncertainty through the different layers (design phases). At each phase, from phase A to phase F, the fidelity of the mathematical model describing each subsystem and component is increased. Thus, it can be considered as a multi-fidelity approach for the design of a complex system affected by epistemic uncertainty. The framework of Dempster-Shafer Theory of Evidence (DST) is used to model epistemic uncertainty. The model is then called Multi-Layer Evidence Network Model (ML-ENM).
\end{abstract}

Keywords: Multy-layer Evidence Network Model, Evidence Theory, Robust Design.

\section{Introduction}

The approaches to the design of engineering systems have been evolving in time at an equal pace with the development of technology and in particular with the increase of computational power. Within the Design by Formula, the first traditional approach in engineering design, the solution is generated by the engineer without the help of any tool and it is based only on the feedback given by the physical prototype. In the Design by Analysis ${ }^{1}$ approach, virtual prototype (software analysis based on numerical methods) gives an important contribution, but still the design process is based on a reductionist approach where subsystems and components are design separately without a particular attention to their interfaces. A system level design, instead, is handled by the Design by Optimisation ${ }^{2}$ with the use of numerical optimisation algorithms integrated with analysis tools. A fundamental improvement is given then by the Design for Reliability and Robustness and more in general Multi-Disciplinary Optimisation (MDO) under Uncertainty $^{3,4}$ that better model a real (engineering) system which is inevitably affected by uncertainty and imprecision. Design Under Uncertainty (DUU) makes designers able to handle higher degree of complexities but, on the other hand, it is particularly challenging due to its high computational cost. If one looks at the different types of uncertainty that a system can be subjected to, two macro-categories can be identified: aleatory uncertainty and epistemic uncertainty. ${ }^{5}$ Aleatory uncertainty is natural randomness which cannot be reduced. Epistemic uncertainty is due to the lack of information or incomplete data. This type of uncertainty is reducible by acquiring more knowledge on the problem. Considering this, a further step forward have been proposed by the authors with Design by Resilience. ${ }^{6}$ Our proposed concept of Resilience Engineering extends and integrates the concepts of Design for Reliability and Design for Robustness and uses the framework of Dempster-Shafer theory of evidence (DST) ${ }^{7}$ to include epistemic uncertainty.

We are here particularly interested in space systems. They are complex systems involving multiple interconnected components and disciplines with complex couplings: payload, structure, thermal analysis, attitude, control, etc. A system level optimal solution cannot be found by optimising the single subsystems independently. Furthermore, the design and optimisation of space systems have to account for epistemic uncertainty, in particular in the early design phase. In fact, knowledge about system and requirements is only acquired incrementally, but 
substantial commitments are made upfront, essentially in the unknown.

Even if the research field is demonstrating to be very active in proposing new and promising methodologies for the DUU of complex systems, space industry, on the other side, has a conservative approach that is based on traditional methods. In fact, the most common and well-established approach to handle uncertainty in space systems engineering is to use safety margins and redundancies. ${ }^{8}$ These traditional methods, however, present two critical problems that affect the result of the design process. There is a lack of an appropriate quantification of uncertainty that brings to an overestimation or an underestimation of the effect of uncertainty (increase in costs and development time or occurrence of undesirable events). There is also a lack of an holistic view on the system performance and evolution.

In this paper, then, we propose a methodological advancement to solve those two problems with specific application to the design of space systems. The novelty is given by a mathematical model, in the form of a multi-layer graph, that simulate the evolution in time of the space system during the design process and is able to quantify and propagate epistemic uncertainty through the different design phases. The model, called Multi-layer Evidence Network Model (ML-ENM) is a generalisation of the Evidence Network Model (ENM) already suggested by the authors. The ENM formulation was first introduced in Ref. ${ }^{9}$ The method was extended in Ref. ${ }^{10}$ to make ENM computationally more efficient. Ref. ${ }^{11}$ introduced a time-dependent reliability measure in the ENM and finally Ref. $^{6}$ introduced the concept of resilience. The ML-ENM allows for a rigorous and fast propagation of epistemic uncertainty ${ }^{6,9-11}$ and gives an holistic view to the whole design process. Each layer represents a different phase in the design process, each node represents a subsystem or a component at a particular level of granularity and each link is a sharing of information.

In particular, this paper proposes a method to propagate uncertainty through the ML-ENM from the last design phase to the first one. Then the system is optimised for robustness with a min-max algorithm. ${ }^{12,13}$ Evidence Theory is applied to quantify uncertainty on the optimal solution. ${ }^{6,9-11}$ It is finally shown that the optimal solution at phase $\mathrm{A}$ is robust against the uncertainty in the next design phases.

\section{Evidence Framework for Epistemic Uncertainty}

As previously stated in Sec.1, the design process of a space system is affected (particularly in the early phases) by epistemic uncertainty that can not be quantified by probability distributions. To model this type of uncertainty we propose the use of DST because it can well capture epistemic uncertainty expressed in the form of expert opinions. ${ }^{7}$

Under the assumptions of independence of the sources of information and uncorrelation of uncertainties, we can define the set $\Theta$ of all the mutually exclusive and collectively exhaustive elementary events (or hypotheses) $\Theta=\left\{\theta_{1}, \theta_{2}, \ldots, \theta_{i}, \ldots, \theta_{|\Theta|}\right\}$. The collection of all non empty subsets of $\Theta$ is the Power Set $2^{\Theta}=(\Theta, \cup)$. One can now assign a probability mass, called basic probability assignment $(b p a)$ to the elements of $2^{\Theta}$. Each element of $2^{\Theta}$ with a non-zero bpa is called a Focal Element $(F E)$ and is represented with the symbol $\gamma$ in the following. The pair $\left\langle\Gamma, b p a_{\Gamma}\right\rangle$ - where $\Gamma \ni \gamma$ and $b p a_{\Gamma} \ni b p a_{\gamma}$ - is called the Body of Evidence.

We can now define the performance index of the system we want to analyse as:

$$
f(\mathbf{d}, \mathbf{u}): D \times U \subseteq \mathbb{R}^{m+n} \rightarrow \mathbb{R}
$$

where $D$ is the design space for the decision or design parameters $\mathbf{d}$, of dimension $m$, and $U=2^{\Theta}$ the event space for the uncertain parameters $\mathbf{u}$, of dimension $n$, that we call the Uncertain Space.

DST measures the influence of uncertainty on the quantity $f$, for a fixed design vector $\mathbf{d}^{*}$, by means of two functions, Belief and Plausibility, that generalise the concept of Probability measure given in classical probability theory. If we are interested in the amount of evidence associated to the event $f(\mathbf{d}, \mathbf{u}) \in \Phi$ we can define

$$
\Omega=\{\mathbf{u} \in U \mid f(\mathbf{d}, \mathbf{u}) \in \Phi\}
$$

as the corresponding set in $U$ and then compute the cumulative Belief and Plausibility associated to that event:

$$
\begin{aligned}
& \operatorname{Bel}(\Omega)=\sum_{\gamma_{i} \subset \Omega, \gamma_{i} \in U} \operatorname{bpa}\left(\gamma_{i}\right), \\
& \operatorname{Pl}(\Omega)=\sum_{\gamma_{i} \cap \Omega \neq 0, \gamma_{i} \in U} \operatorname{bpa}\left(\gamma_{i}\right) .
\end{aligned}
$$

From Eqs. (3) and (4) we can state that the belief in the realisation of the event $f(x) \in \Phi$ is the sum of the bpa of all the FEs totally included in $\Omega$, while the Plausibility is the sum of all the FEs that have a non-null intersection with $\Omega$. More details about the DST can be found in Ref.

\section{Evidence-Based Robust Optimisation}

This section explains the approach we use to incorporate epistemic uncertainty in the optimisation process and to design the system for robustness.

Given the performance index $f$ in (1), Evidence-Based Robust Optimisation aims at finding the decision vector $\mathbf{d}^{*}$ that maximises the Belief in statement (2), given a body of evidence, and optimises the set $\Phi$. The concept was introduced by the authors in Ref. ${ }^{14}$ and extended in Ref. ${ }^{15}$

If one is interested in the minimisation of $f$ under the satisfaction of a constraint function $C \leq v_{C}$, Eq. (2) translates in the following two sets of uncertain parameters:

$$
\Omega=\{\mathbf{u} \in U \mid f(\mathbf{d}, \mathbf{u}) \leq v\}
$$




$$
\Omega_{C}=\left\{\mathbf{u} \in U \mid C(\mathbf{d}, \mathbf{u}) \leq v_{C}\right\}
$$

where we want to minimise $f$ and maximise the belief in the statement (5) while maintaining an hard condition on the constraint satisfaction:

$$
\begin{aligned}
& \max _{\mathbf{d} \in D} \operatorname{Bel}(f(\mathbf{d}, \mathbf{u}) \leq v) \\
& \min _{v \in \mathbb{R}} v \\
& \operatorname{Bel}\left(C(\mathbf{d}, \mathbf{u}) \leq v_{C}\right)>1-\varepsilon
\end{aligned}
$$

Problem (7) requires the evaluation of the belief curve for both the functions $f$ and $C$ and it becomes easily intractable. In fact there is a dependence of the belief to the design vector $\mathbf{d}$ and the thresholds $v$ and $v_{C}$ thus for each new value of $\mathbf{d}, v$ and $v_{C}$ the belief has to be revalued. Furthermore the exact belief reconstruction requires a number of maximisations equal to the number of $F E$ s and this number increases exponentially with the problem dimension.

Among all vectors $\mathbf{d}$ that solve problem (7) the most critical one, $\mathbf{d}^{*}$, corresponds to the minimum values of $v$ and $v_{C}$ such that $\operatorname{Bel}(f(\mathbf{d}, \mathbf{u}))$ is maximum and $\left.\operatorname{Bel}\left(C(\mathbf{d}, \mathbf{u}) \leq v_{C}\right)\right)=1$. We call the search for $\mathbf{d}^{*}$, worst-case scenario optimisation and it can be formulated as the deterministic min-max optimisation problem: ${ }^{3}$

$$
\begin{aligned}
& \min _{\mathbf{d} \in D} \max _{\mathbf{u} \in U} f(\mathbf{d}, \mathbf{u}) \\
& \text { s.t. } \\
& \forall \mathbf{u} \in U: \quad C(\mathbf{d}, \mathbf{u}) \leq 0 .
\end{aligned}
$$

Solving for the worst-case scenario makes the optimisation problem independent of the uncertainty quantification method, has a complexity that is independent of the number of $F E$ s and does not require any particular assumption on the constraint functions.

\section{Space Systems Project Life Cycle}

Space missions are complex and requires time to be designed and implemented. The development process, in fact, requires several years from early conception to launch and commissioning. Also it involves different players who have different goals: end user or costumer, operators, scientists, developer and sponsor. In order to decompose the whole design process in smaller and more manageable pieces, the life cycle of a space mission traditionally proceeds through four main phases: the concept exploration phase, that broadly defines the space mission and its components, the detailed development phase, that defines more precisely system's components, the production and deployment phase, that constructs and launches the system, the operations and support phase, that daily supports the mission and brings it to its end of life. ${ }^{16}$ Depending on the mission's sponsor (NASA, ESA, DoD, commercial enterprise, ...), these phases are further divided and labelled differently. For example, NASA divides the project life cycle in seven incremental steps: ${ }^{17,18}$ Pre-Phase A (concept study), Phase A (concept and tecnolongy development), Phase $B$ (Preliminary Design and Technology Completion), Phase $C$ (Final Design and Fabrication), Phase D (System Assembly, Integration and Test, Launch), Phase E (Operations and Sustainment) and Phase F (Closeout). Phases A, B, ..., F are separated by Key Decision Points (KDPs) that are events in which the authority, based on the progress state, the achieved results, the requirements and the budget, approves or rejects the project with a "go" or "no go" decision.

An alternative grouping is represented by the following macro-phases.

\subsection{Pre-Formulation}

This phase is not part of the project life-cycle. It is nevertheless of fundamental importance. Feasibility and desirability are here preferred to optimality. Engineers are interested in a broad analysis of risks, cost, feasibility. A variety of possible scenarios and ideas are analysed.

\subsection{Formulation}

During the formulation, that includes phase A and phase $\mathrm{B}$, the full range of implementation options are explored and finally a promising design concept is proposed. The formulation includes the development of the system architecture. Preliminary mission and design are finalised thanks to trades between safety, risk, performance and cost. Finally the project plan is prepared for the next phase.

\subsection{Implementation}

The project implementation consists of phases C, D, E and F. During phase $\mathrm{C}$, there is the completion of the final system design, the fabrication and the test of components, assemblies and subsystems. Phase D, instead, includes the system assembly integration and test, the pre-launch activities, the launch, on-orbit check out, and the initial operations. Phase E controls the operation during the mission life-time. Finally, phase F concludes.

\section{Multi-layer Evidence Network Model (ML-ENM)}

This section introduces the concept of ML-ENM that can be used to quantify and propagate epistemic uncertainty through the complex system and the different phases of the whole design process.

ML-ENM generalises the ENM that has been presented in Refs. ${ }^{6,-11}$ ENM is a framework for a decomposition procedure that evaluate Belief and Plausibility curves with a computational cost that is polynomial and not exponential with the problem dimension.

Although a network representation for complex systems is a common approach in MDO, the presented (ML-)ENM gives new and important advantages with respect to the commonly used Design Structure Matrix (DSM). ${ }^{19}$ In particular, within (ML-)ENM the correlations between nodes are represented by scalar values that model in a compact way the influence of many uncertain parameters and weight the different links. Also (ML-)ENM allows for an easier representation of sub-networks and cluster. 
ML-ENM is a multi-layer network where each layer represents a different phase in the design process, each node represents a subsystem or a component at a specific level of granularity and each link is a sharing of information. As the design process proceeds from pre-phase A to phase D, an increasing level of detail is needed in the analysis, the focus is shifted from the subsystem level to the component level, more precise mathematical models are implemented and the number of nodes increases. On the other side, studying how real projects evolve, there is a high level of confidence that between a phase and the following one, unforeseen circumstances require a modification of the design requirements and goals. Furthermore, different players collaborate in the project and, usually, a good communication between them is not an easy task. Based on the results of the single design phase and on the uncertainty on the whole process' evolution, the designers take decisions that bring them to the next phase and that will drive the design process.

Looking at Fig. 5, for example, during phase A three subsystems are considered and optimised. During phase B the number of considered components is increased. The point is that the number of sub-divisions and the types of the components in phase B depend on the designers' choices and each decision brings the design process to a different final solution. Also, the number of possible final configurations increases exponentially with the number of layers and possible choice that can be selected between each couple of layers.
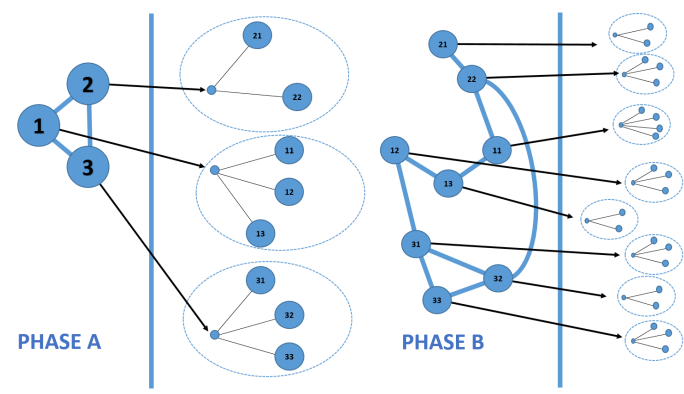

Figure 1: Evolution of the ENM between phase A and B: each node in phase $\mathrm{A}$ is decomposed in two or more nodes in phase B. The number of nodes and the mathematical model associated to them depend on the designers' choices. The process is then repeated for the next phases.

More formally, a ML-ENM with $N_{L}$ layers is a pair $(\mathrm{G}, \mathrm{C})$ where $\mathrm{G}=\left\{G_{\alpha} ; \alpha \in\left\{1, . ., N_{L}\right\}\right\}$ is a family of directed and weighted graphs $G_{\alpha}=\left(X_{\alpha}, E_{\alpha}\right)$ and $C=\left\{E_{\alpha \beta} \subset X_{\alpha} \times X_{\beta} ; \alpha, \beta \in\left\{1,2, \ldots, N_{L}\right\}, \beta=\alpha+1\right\}$ is the set of interconnections between nodes of different layers. The intralayer links in $E_{\alpha}$ represents the sharing of information between subsystems and components of the space system (complex system). The interlayer links in $E_{\alpha \beta}$ model the decision process tree between different design phases.

Design $\mathbf{d}$ and uncertain $\mathbf{u}$ vectors are decomposed in two components: $\mathbf{d}=\left[\mathbf{d}^{d}, \mathbf{d}^{s}\right]^{T}$ and $\mathbf{u}=\left[\mathbf{u}^{d}, \mathbf{u}^{s}\right]^{T}$ where the former $\left(\left[\mathbf{d}^{d}, \mathbf{u}^{d}\right]^{T}\right)$ are related to the interlayer decision process, between a layer and the next one, and the latter $\left(\left[\mathbf{d}^{S}, \mathbf{u}^{S}\right]^{T}\right)$ describe the intralayer physical model of the space system at a particular level of resolution.

At each layer $\alpha \in\{$ pre- $A, B, \ldots, F\}$ of the ML-ENM, the performance index can be defined as:

$$
f^{\alpha}(\mathbf{d}, \mathbf{u})=\sum_{i=1}^{N} g_{i}^{\alpha}\left(\mathbf{d}_{i}^{\alpha s}, \mathbf{u}_{i}^{\alpha s}, \varphi_{i}^{\alpha s}\left(\mathbf{d}_{i}^{\alpha s}, \mathbf{u}_{i}^{\alpha s}, \mathbf{d}_{i j}^{\alpha s}, \mathbf{u}_{i j}^{\alpha s}\right)\right),
$$

In Eq. (9) $N$ is the number of nodes of the network in layer $\alpha$ and $\varphi_{i}^{\alpha}\left(\mathbf{d}^{\alpha}, \mathbf{u}_{i}^{\alpha}, \mathbf{d}_{i j}^{\alpha s}, \mathbf{u}_{i j}^{\alpha}\right)$ is the vector of scalar exchange functions $\varphi_{i j}^{\alpha}\left(\mathbf{d}^{\alpha}, \mathbf{u}_{i}^{\alpha}, \mathbf{d}_{i j}^{\alpha s}, \mathbf{u}_{i j}^{\alpha}\right)$ that represent the input/output of the nodes, with $j \in J_{i}^{\alpha}$, and $J_{i}^{\alpha}$ the set of indexes of nodes connected to the $i$-th node of that layer. Eq. (9) decomposes the uncertain components $\mathbf{u}^{s}$ in two categories: the uncoupled components $\mathbf{u}_{i}^{\alpha s}$ that affect only subsystem $i$, and the coupled variables $\mathbf{u}_{i j}^{\alpha s}$ shared among subsystem $i$ and one or more subsystems $j$.

\section{Problem Formulation}

The ML-ENM has been here applied to the design for robustness of a spacecraft through the phases A, B and $\mathrm{C}$ (pre-phase $\mathrm{A}$ is considered in the figures for clarity). Each node of the ML-ENM is associated to a mathematical function modelling a subsystem or a component. Their list and the classification between the different phases is presented in Tab. 1. The quantity of interest is the overall mass of the satellite and it is given by the sum of the masses of all the subsystems (phase A) or components (phase B and $\mathrm{C}$ ). The network can be visualised in Figs. 2, 3, 4 and 5: the nodes correspond to the models of the system (node 1 at pre-phase A), sub-systems (nodes 2-6 at phase A) and components (nodes 7-18 at phase B and nodes 19-30 at phase C). The links, instead, correspond to their intra-layer and inter-layer connection. In particular coloured arrows define inter-layer (hierarchical) dependencies while grey lines indicate intra-layer dependencies. Red lines show the dependence of nodes at layer $\mathrm{A}$ from the node at layer pre-A (pre- $A \rightarrow A$ ), yellow lines show the dependence of nodes at layer $\mathrm{B}$ from nodes at layer $\mathrm{A}(A \rightarrow B)$ and purple lines of nodes at layer $\mathrm{C}$ from layer $\mathrm{B}(B \rightarrow C)$. Each node in a generic layer, in fact, can be decomposed in two or more nodes in the next layer. Furthermore, the number of parameters and the complexity increase through the process as Tab. 2 shows. Gray lines instead represent couplings between nodes in the same layer $\alpha$ through the linking functions $\varphi_{i}^{\alpha}$ as in Eq. 9.

\section{Method}

For the defined ML-ENM, the Body of Evidence presented in Sec. 2 can be populated at the last phase of the ML-ENM, 
Table 1: ML-ENM nodes

\begin{tabular}{lc}
\hline Node & Pre-Phase A \\
\hline 1 & Spacecraft \\
& Phase A \\
& \\
2 & Attitude and Orbit Control (AOCS) \\
3 & Payload \\
5 & Power \\
6 & Thermal \\
& Telemetry and Telecommand (TTC) \\
& Phase B
\end{tabular}

Table 2: Model dimention

\begin{tabular}{l|ll|ll}
\hline \multirow{2}{*}{ Phase } & \multicolumn{2}{|c|}{ Path 1 } & \multicolumn{2}{c}{ Path 2 } \\
& $\operatorname{dim}_{d}$ & $\operatorname{dim}_{u}$ & $\operatorname{dim}_{d}$ & $\operatorname{dim}_{u}$ \\
\hline A & 6 & 16 & 6 & 17 \\
B & 13 & 35 & 14 & 34 \\
C & 21 & 43 & 21 & 47 \\
\hline
\end{tabular}

\section{ML-ENM}

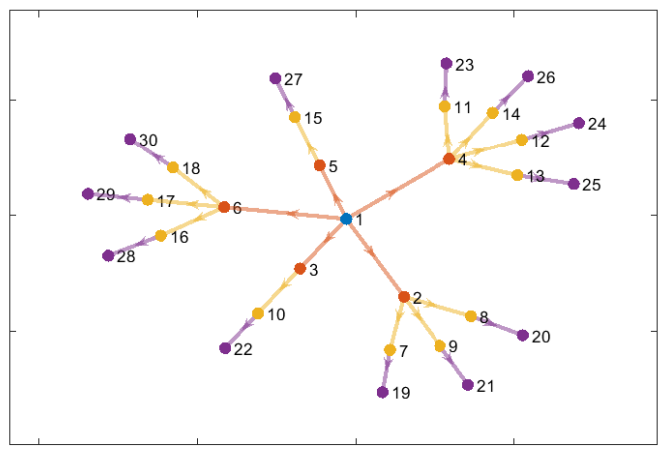

\section{Magnetorquers}

Thrusters

Reaction Wheels

Payload

Batteries

Harness

Power Conditioning and Distribution Unit (pcdu)

Solar Array

Thermal

Antenna

Radio Frequency Distribution Network (rfdn) Transponder

\section{Phase C}

Magnetorquers

Thrusters

Reaction Wheels

Payload

Batteries

Harness

Power Conditioning and Distribution Unit (pcdu)

Solar Array

Thermal

Antenna

Radio Frequency Distribution Network (rfdn)

Transponder
Figure 2: $2 \mathrm{D}$ representation of the design process as a decision tree. The phases (A, B and C) are indicated with different colours. Subsystem's and component's models are represented as nodes.

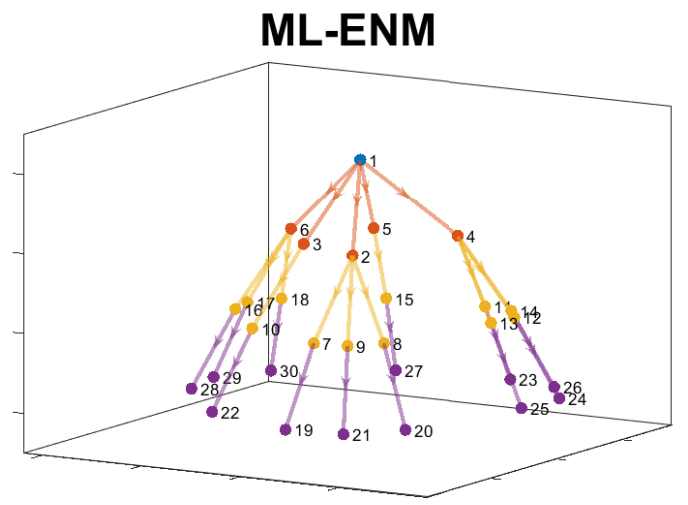

Figure 3: 3D representation of the design process as a tree.

here phase $\mathrm{C}$, by a process of knowledge elicitation. For the proposed application, available data from previous publications has been used. ${ }^{9}$

In this example there are only two possible paths that the design process can explore from phase A to phase C. They correspond to the choice between node 7 (Magnetorquers) and 8 (Thruster) at phase B. The choice brings, respectively, to node 19 and 20 at layer $\mathrm{C}$.

For each chosen path the uncertainty structure at phase $\mathrm{C}$ is propagated back to phase A exploiting the inter-layer dependencies $\mathrm{A} \rightarrow \mathrm{B}$ and $\mathrm{B} \rightarrow \mathrm{C}$. A minimisation and $\mathrm{a}$ 


\section{ML-ENM}

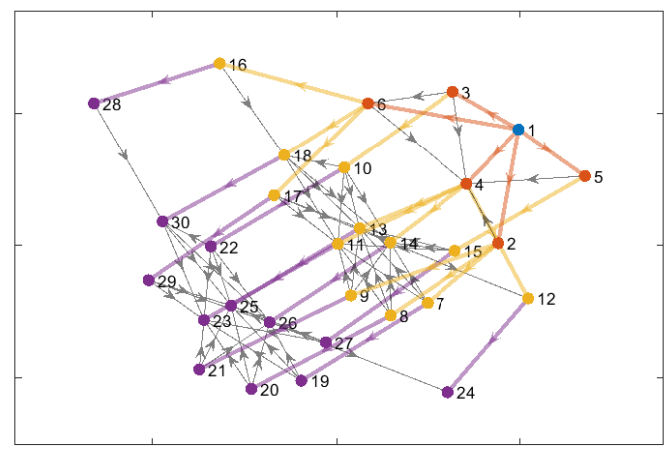

Figure 4: Representation of the design process as a graph. Coloured arrows define inter-layer dependencies while grey lines indicate intra-layer dependencies within the same design phase.

\section{ML-ENM}

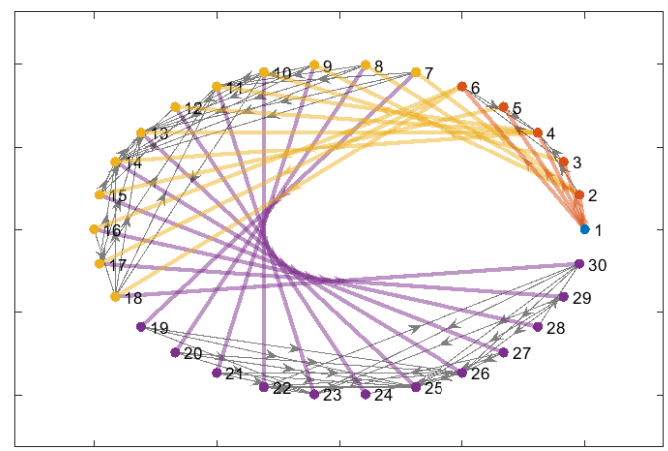

Figure 5: Circular representation of the ML-ENM with both inter-layer and intra-layer dependencies.

maximisation have been run to reconstruct the lower and upper bounds of each uncertain parameter at layer $\alpha$ that incorporate two or more parameters of the layer $\alpha+1$. In this manner, the reconstructed Body of Evidence at phase $A$ incorporates the uncertainty that affect the more complex and detailed models at phase B and C.

Then, the system is optimised for robustness at phase $A$. In particular, the min-max algorithm is used to evaluate the optimal design vector $\mathbf{d}_{A}^{*}$. For more details about the method please refer to Refs. ${ }^{12,13}$

For the evaluated optimal design solution $\mathbf{d}_{A}^{*}$, the decomposition approach presented in Refs. ${ }^{6,9-11}$ has been applied to the ML-ENM in order to propagate uncertainty through the spacecraft model and reconstruct a good approximation of the belief curve with a fraction of the computational cost required for the exact one (Fig. 12).

The effect of uncertainty at phases B and C is finally analysed in correspondence with the robust design solution
$\mathbf{d}_{A}^{*}$.

\section{Results}

Considering the first path in the ML-ENM (node 7 at phase $\mathrm{B}$ and node 19 at phase $\mathrm{C}$ ), the worst case optimal solution $\left(\mathbf{d}_{\mathbf{1}}^{*}, \mathbf{u}_{\mathbf{1}}^{*}\right)$ gives a mass of $166.43 \mathrm{~kg}$. Figs. 6, 7 and 8 show the effect of uncertainty at phases A, B and C for the fixed $\mathbf{d}_{\mathbf{1}}^{*}$. The second path (node 8 at phase $\mathrm{B}$ and node 20 at phase $\mathrm{C}$ ) brings to the robust solution $\left(\mathbf{d}_{\mathbf{2}}^{*}, \mathbf{u}_{\mathbf{2}}^{*}\right)$ with a corresponding mass of $230.12 \mathrm{~kg}$. Figs. 9, 10 and 11 show the effect of uncertainty at phases $\mathrm{A}, \mathrm{B}$ and $\mathrm{C}$ for the fixed $\mathbf{d}_{\mathbf{2}}^{*}$. In particular, Figs. 6 and 9 concern the system level (the whole mass of the satellite), Figs. 7 and 10 the sub-systems level and Figs. 8 and 11 the components level. The boxplots have been evaluated with a Monte Carlo simulation over the uncertain space with $10^{6}$ function evaluation. For each box a maximisation and a minimisation have been run in order to be sure that the boxes include all the possible values of the mass for the given uncertainty structure. These figures show that the spacecraft model at phase A with the back-propagation of uncertainty, incorporates for the chosen path all the uncertainty in phases $\mathrm{B}$ and $\mathrm{C}$. The worst case optimal solution $\mathbf{d}^{*}$ at phase $\mathrm{A}$, then, results to be robust through the design process.

For $\mathbf{d}_{\mathbf{1}}^{*}$, finally, the decomposition approach has been applied to the ML-ENM and Fig. 12 presents the reconstructed belief curve. The decomposition method allows to fast and good evaluation of the belief as demonstrated in Ref. ${ }^{10}$ In this problem, in fact, the exact evaluation of the belief curves require 65536 maximisations (one for each focal element). Instead, the curve in Fig. 12 has been evaluated with 234 maximisations $(0.36 \%)$.

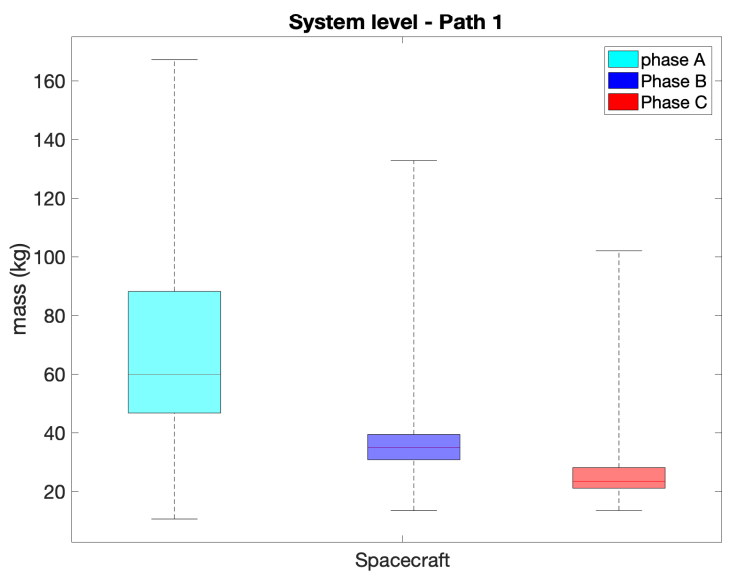

Figure 6: Effect of uncertainty at the system's level in phases $\mathrm{A}, \mathrm{B}$ and $\mathrm{C}$ for the first considered path. The design vector is fixed at the optimal solution.

\section{Conclusion}

This paper proposes a new approach for the design process of a space system affected by epistemic uncertainty. The 


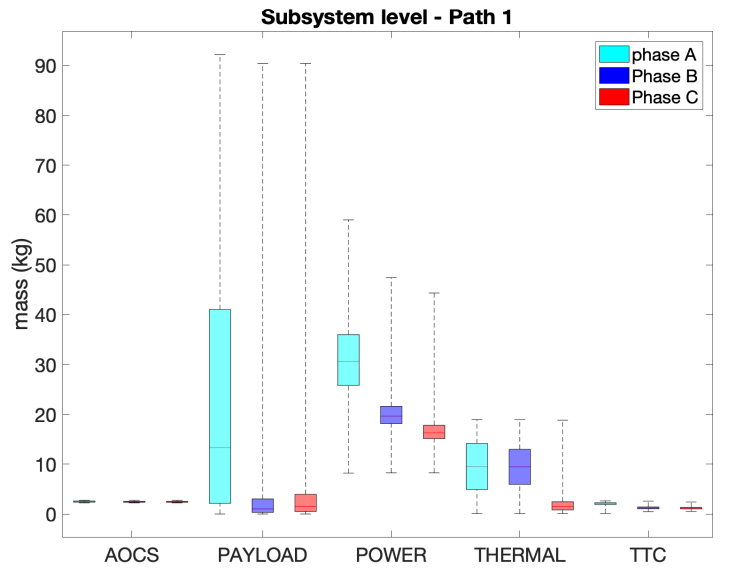

Figure 7: Effect of uncertainty at the sub-system's level in phases $\mathrm{A}, \mathrm{B}$ and $\mathrm{C}$ for the first considered path. The design vector is fixed at the optimal solution.

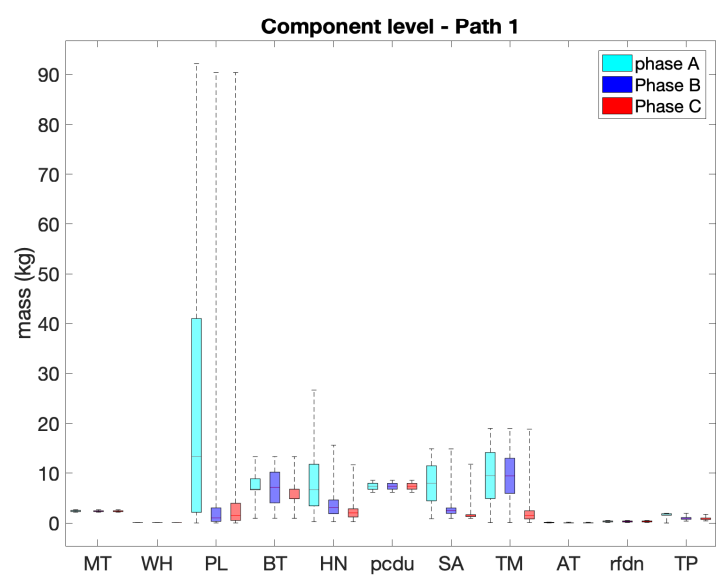

Figure 8: Effect of uncertainty at the component's level in phases A, B and C for the first considered path. The design vector is fixed at the optimal solution.

main novelty is given by the use of the ML-ENM to quantify and propagate uncertainty between different phases of the design process. ML-ENM is a multi-layer network representation of the complex system where each layer takes into account the couplings between subsystems (or components) at a particular design phase. The evolution of the design process is then modelled by the sequence of layers.

It is here presented a method for the definition of uncertainty at the first phase (phase A) of the process such that the optimal solution at that phase is robust against the uncertainty in the following phases.

The method is applied to the design of a space system. The model is optimised for robustness and finally a decomposition methodology is applied to the network in

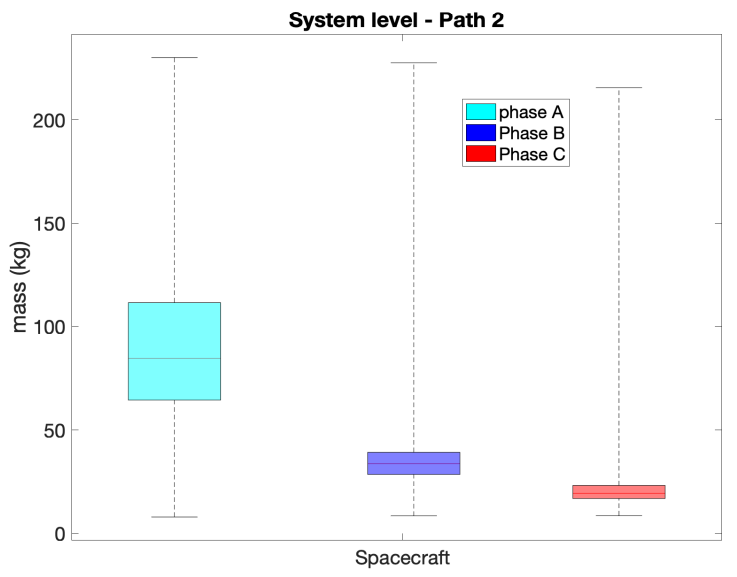

Figure 9: Effect of uncertainty at the system's level in phases $\mathrm{A}, \mathrm{B}$ and $\mathrm{C}$ for the second considered path. The design vector is fixed at the optimal solution.

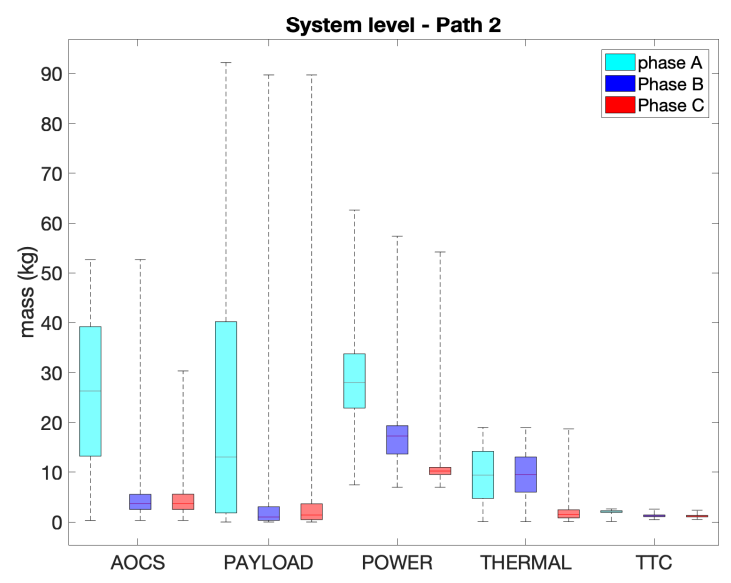

Figure 10: Effect of uncertainty at the sub-system's level in phases $\mathrm{A}, \mathrm{B}$ and $\mathrm{C}$ for the second considered path. The design vector is fixed at the optimal solution.

order to reduce the computational cost of the epistemic uncertainty propagation and the belief reconstruction with the use of Evidence Theory.

It has be shown that the optimal design solution at phase A defined in such a way, is robust against the propagation of uncertainty through the design process.

\section{Acknowledgement}

The work in this paper was supported by the H2020-MSCA-ITN-2016 UTOPIAE, grant agreement 722734.

\section{References}

[1] Pedersen, P. and Laursen, C. L. Design for Minimum Stress Concentration by Finite Elements and Linear 


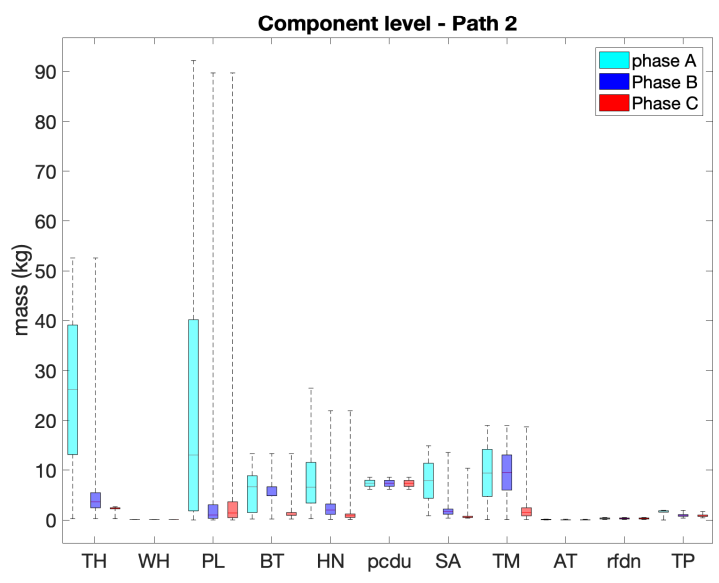

Figure 11: Effect of uncertainty at the component's level in phases $\mathrm{A}, \mathrm{B}$ and $\mathrm{C}$ for the second considered path. The design vector is fixed at the optimal solution.

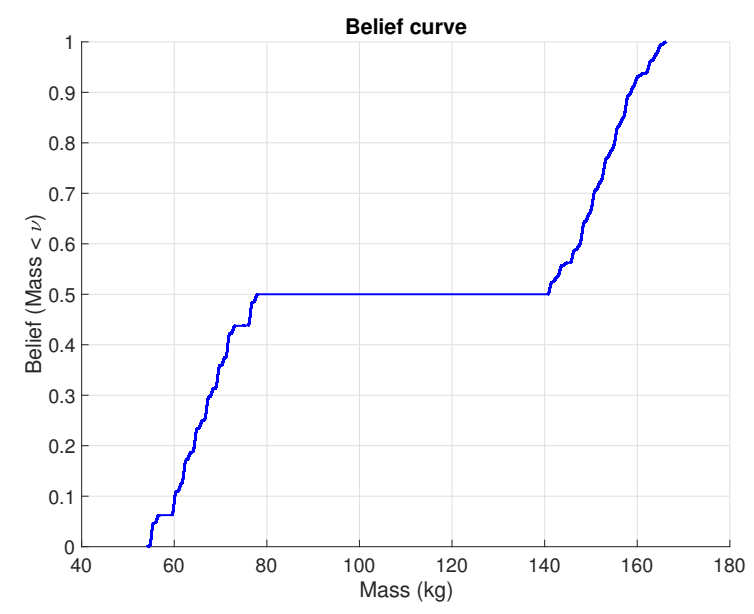

Figure 12: Cumulative Belief curve of the optimal worst case solution at phase A for the first considered path.

Programming. Journal of Structural Mechanics 10(4), 375-391 jan (1982).

[2] Nicolich, M. and Cassio, G. System Models Simulation Process Manangement and Collaborative Multidisciplinary Optimization. In INCOSE Italian Chapter Conference on Systems Engineering (CIISE2014) (, Rome, Italy, 2014).

[3] Beyer, H.-g. and Sendhoff, B. Robust optimisation: A comprehensive survey. Comput. Methods Appl. Mech. Engrg. 196, 3190-3218 (2007).

[4] Zio, E. Reliability engineering: Old problems and new challenges. Reliability Engineering and System Safety 94(2), 125-141 (2009).
[5] Helton, J. C., Johnson, J. D., Oberkampf, W. L., and Sallaberry, C. J. Representation of analysis results involving aleatory and epistemic uncertainty. International Journal of General Systems 39(6), 605-646 (2010).

[6] Filippi, G., Vasile, M., Krpelik, D., Z., K., Marchi, M., and C., P. Space Systems Resilience Optimisation Under Epistemic Uncertainty. Acta Astronautica (2019).

[7] Shafer, G. A mathematical theory of evidence. Princeton University Press, (1976).

[8] Division, S. Space engineering: Engineering design model data exchange (CDF). Technical Report ECSS-TM-E-10-25A 20 October 2010 First, (2010).

[9] Alicino, S. and Vasile, M. Evidence-based preliminary design of spacecraft. In $6^{\text {th }}$ International Conference on Systems \& Concurrent Engineering for Space Applications (, Vaihingen Campus, University of Stuttgart, Germany, 08-10 October 2014).

[10] Vasile, M., Filippi, G., Ortega, C., and Riccardi, A. Fast belief estimation in evidence network models. In EUROGEN (, Madrid, 13-15 September 2017).

[11] Filippi, G., Marchi, M., Vasile, M., and Vercesi, P. Evidence-Based Robust Optimisation of Space Systems with Evidence Network Models. In 2018 IEEE Congress on Evolutionary Computation (CEC), Rio de Janeiro, 1-8. IEEE, jul (2018).

[12] Vasile, M. On the solution of min-max problems in robust optimization. In The EVOLVE International Conference (, Jian-Guo Hotel, China, 1-4 July 2014).

[13] Filippi, G. and Vasile, M. A memetic approach to the solution of constrained min-max problems. (IEEE congress on evolutionary computation, Wellington, New Zealand, 10-13 June 2019).

[14] Vasile, M. Robust mission design through evidence theory and multiagent collaborative search. Annals of the New York Academy of Sciences 1065, 152-173 (2005).

[15] Alicino, S. and Vasile, M. An evolutionary approach to the solution of multi-objective min-max problems in evidence-based robust optimization. In Proceedings of the 2014 IEEE Congress on Evolutionary Computation, CEC 2014, (2014).

[16] Wertz, J. and Larson, W. Space Mission Analysis and Design. 3rd ed., Microcosm Press, (1999). 
[17] NASA NPR7120.5. NPR 7120 . 5, NASA Space Flight Program and Project Management Handbook. NASA's Procedural Requirements (February) (2010).

[18] Hirshorn, S. NASA System Engineering Handbook SP-2016-6105 Rev2. , 297 (2016).

[19] Hu, X., Chen, X., Lattarulo, V., and Parks, G. T. Multidisciplinary Optimization Under High-Dimensional Uncertainty for Small Satellite System Design. (2015). 